\title{
Plastic rearrangements in colloidal gels investigated by LAOS and LS-Echo
}

\author{
M. Laurati ${ }^{\text {a) }}$ and S. U. Egelhaaf \\ Condensed Matter Physics Laboratory, Heinrich Heine University, \\ Universitätsstr. 1, Düsseldorf 40225, Germany \\ G. Petekidis \\ IESL-FORTH and Department of Materials Science and Technology, \\ University of Crete, Heraklion, Greece
}

(Received 3 January 2014; final revision received 9 April 2014;

published 26 August 2014)

\begin{abstract}
Synopsis
We investigate the yielding behavior of colloid-polymer gels with intermediate volume fraction, which are subjected to large amplitude oscillatory shear, using rheology and light scattering echo (LS-Echo). Particular attention is given to the anharmonic contributions to the stress response and the characteristic timescale and extent of plastic rearrangements. Yielding is already observed at small strain amplitudes $\gamma_{0} \approx 1 \%$, where the network of interconnected clusters starts to break up and irreversible particle rearrangements are first observed. However, only at considerably larger strain amplitudes, $\gamma_{0} \gtrsim 100 \%$, the network is completely disrupted and small clusters or individual particles flow. This complex yielding behavior is reflected in different regimes of in-cycle yielding, which were extracted from the analysis of the anharmonic contributions to the stress. In the range of strain amplitudes where bond breaking starts, in-cycle strain hardening and shear thickening are observed, with the strain hardening possibly caused by the shear-induced formation of more compact clusters. When the network is broken down, in-cycle shear thinning is observed with increasing strain amplitude, but still together with strain hardening. Both, the elastic and viscous nonlinear contributions, decrease with increasing strain amplitude, indicating the progressive fluidization of the system, consistent with the faster plastic rearrangements observed in LS-Echo. Two-distinct frequency-dependent regimes for the initial yielding at small strains are present: At small to moderate oscillation frequencies, the first yield strain increases with increasing frequency, while it decreases at large frequencies. This might be associated with the timescale for bond breaking, namely, the Brownian diffusion time at small frequencies and the inverse of the oscillation frequency at large frequencies. (C) 2014 The Society of Rheology. [http://dx.doi.org/10.1122/1.4872059]
\end{abstract}

\section{INTRODUCTION}

Many household products, like toothpaste, creams, hair-care products, or food, like jellies and gelatins, is typically in an amorphous solidlike state, which is known as a gel

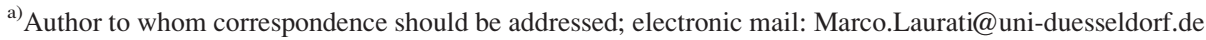


(Encyclopedia britannica ${ }^{1}$ ). The solidlike nature of a gel arises from a microscopic network structure, which is able to sustain stress, $\sigma$, up to a certain limit, which is defined as the yield stress $\sigma_{y}$ [Zaccarelli (2007)]. The network structure of gels can either be of chemical or of physical origin [Zaccarelli (2007)]. In chemical gels, the network is formed by irreversible chemical reactions (which can be induced by external stimuli), while in physical gels attractions between individual particles lead to interparticle "bonds" with bond energies of the order of the thermal energy $k_{\mathrm{B}} T$; in this case, bonding hence is reversible. Colloid-polymer mixtures have been intensively investigated as a model system of physical gels [Poon (2002); Zaccarelli (2007)]. The nonadsorbing polymers induce a so-called depletion effect [Asakura and Oosawa (1954)] leading to effective attractions between the colloidal particles. Their range and magnitude depend on the polymer-to-colloid size ratio and the polymer concentration, respectively. The effective attractive potential, and hence the polymer size and concentration, together with the colloid volume fraction control gel formation and structure. Gels with different structures are observed: From networks of chainlike aggregates at small volume fractions and/or large attractions to networks of more compact globular clusters at larger volume fractions and moderate attractions [Varadan and Solomon (2003); Shah et al. (2003a); Dibble et al. (2006); Laurati et al. (2009); Furst and Pantina (2007); Lee and Furst (2008)]. Gelation is driven by arrested phase separation at small volume fractions [Verduin and Dhont (1995); Verhaegh et al. (1997); Zaccarelli (2007); Lu et al. (2008)], while percolation [Eberle et al. (2011)] and dynamical arrest [Bergenholtz et al. (2003); Shah et al. (2003b); Laurati et al. (2009)] induce gelation at moderate and large volume fractions, respectively. It has recently been proposed that gelation in systems with short-range attractions might result from rigidity percolation of a dynamic network with an average number of bonds of 2.4 [Valadez-Pérez et al. (2013)].

When shear is applied to these gels, their weak solid nature becomes apparent. In the linear viscoelastic regime, they show a solidlike response [Shah et al. (2003c); Laurati et al. (2009); Koumakis and Petekidis (2011)] with the magnitude of the elastic modulus depending on the structural organization and its degree of heterogeneity [Zaccone and Del Gado (2009)]. In the nonlinear regime, they yield at relatively small stresses and strains in a multistep process involving a progressive disruption of the gel network [Colombo et al. (2013)], which was observed under oscillatory and continuous shear [Laurati et al. (2011); Koumakis and Petekidis (2011)]. At strain amplitudes comparable to the range of the attraction, the bonds connecting different clusters in the network break. Upon increasing the strain amplitude, clusters start to be rearranged and successively broken down. Recent findings indicate that the final state of the gel after shear depends on the applied shear rate and the initial structure [Koumakis (2011); Koumakis et al. (2013b)].

The knowledge on the yielding behavior of colloid-polymer gels profited from large amplitude oscillatory shear (LAOS) measurements, and an analysis in terms of the viscoelastic moduli which assumes a linear stress-strain relation also in the nonlinear regime, i.e., neglects anharmonic contributions to the stress response. However, if this information is exploited, a more detailed picture of the yielding mechanisms may be achieved. In particular, based on the anharmonic contributions, the onset of yielding can be determined and strain hardening/softening and shear thickening/thinning responses within a cycle of deformation can be distinguished [Neidhofer et al. (2003); Ewoldt et al. (2008); Poulos et al. (2013)].

${ }^{1}$ Encyclopedia britannica, http://www.britannica.com/eb/article-9036313/gel, 2007. 
Moreover, the characteristic timescales of plastic rearrangements, which lead to a restructuring of the gel, have not been studied and are therefore unknown. Using echodiffusing wave spectroscopy (Echo-DWS) or light scattering echo (LS-Echo) [Hebraud et al. (1997); Höler et al. (1997); Petekidis et al. (2002b)], the decorrelation between light scattered in the initial configuration, $t=0$, and at multiples of the oscillation period, $t=m T$, can be measured and the degree of plastic deformation and the corresponding timescales deduced (Sec. II B). Furthermore, the decorrelation of the first echo peak at $t=T$ provides an estimate for the onset of shear-induced plastic rearrangements [Petekidis et al. (2002a)]. In hard-sphere glasses, LS-Echo indicates that yielding is linked with the onset of shear-induced particle rearrangements. Furthermore, at larger oscillation frequencies, smaller rearrangements were observed, which have been attributed to the fact that, with increasing frequency, Brownian motion can contribute less to the restructuring [Petekidis et al. (2002a); Petekidis et al. (2003)].

Here, we investigate the response of colloid-polymer gels with an intermediate volume fraction to LAOS for different attraction strengths and oscillation frequencies. We investigate yielding based on the complete stress response data, thus including the anharmonic contributions and hence beyond the average response considered previously [Laurati et al. (2011); Koumakis and Petekidis (2011)]. The LAOS data are analyzed using several methods (Sec. II A), and the obtained results (Sec. IV C) are compared to the linear viscoelasticity (Sec. IV A) and the average yielding behavior (Sec. IV B). We also present results obtained by LS-Echo, especially the characteristic timescales for shear-induced restructuring and the onset of plastic irreversible rearrangements (Sec. IV D).

\section{DATA ANALYSIS METHODS}

\section{A. LAOS}

LAOS measurements have become popular during the last decade as a method to investigate the nonlinear viscoelastic response of a system beyond the first harmonic approximation, which is the standard output of rheometers. Higher order harmonic contributions are accessed by collecting the full time-dependent stress signal during the application of a sinusoidal strain with amplitude $\gamma_{0}$ and frequency $\omega$. The nonlinear contributions in the signal can be extracted following different approaches [Hyun et al. (2011)].

The most common approach is a Fourier transform (FT) analysis. The periodic signal is expressed as a Fourier series [Wilhelm et al. (1998)]. The magnitude of the nonlinearity can be quantified by the ratio of the amplitude of the higher harmonics and the one of the fundamental first harmonic, as shown for many different systems [Wilhelm et al. (1999); Schlatter et al. (2005); Daniel et al. (2001); Langela et al. (2002); Le Grand and Petekidis (2008); Carrier and Petekidis (2009)]. Using this approach, it is difficult to establish a direct relation between the nonlinear response and its physical origin.

In a different approach [Ewoldt et al. (2008)], the total stress is decomposed into elastic, $\sigma^{\prime}$, and viscous, $\sigma^{\prime \prime}$, stresses, as previously proposed [Cho et al. (2005)]. They are then expressed as Chebyshev polynomials

$$
\begin{aligned}
\sigma^{\prime}(x) & =\gamma_{0} \sum_{n: \text { odd }} e_{n}\left(\omega, \gamma_{0}\right) T_{n}(x), \\
\sigma^{\prime \prime}(y) & =\dot{\gamma}_{0} \sum_{n: \text { odd }} v_{n}\left(\omega, \gamma_{0}\right) T_{n}(y),
\end{aligned}
$$


where $T_{n}(\mathrm{)})$ is the $n t h$-order Chebyshev polynomial of the first kind, $x=\gamma(t) / \gamma_{0}$ and $y=\dot{\gamma}(t) / \dot{\gamma}_{0}$, with $\dot{\gamma}_{0}$ the maximum in-cycle shear rate, and $e_{n}\left(\omega, \gamma_{0}\right)$ and $v_{n}\left(\omega, \gamma_{0}\right)$ are the elastic and viscous Chebyshev coefficients of order $n$, respectively. The third-order coefficients, $e_{3}$ and $v_{3}$, are used to classify the response in terms of shear thickening/thinning $\left(v_{3}>0\right.$ and $v_{3}<0$, respectively) and strain hardening/softening $\left(e_{3}>0\right.$ and $e_{3}<0$, respectively). Ewoldt et al. also defined dimensionless indexes of nonlinearity

$$
\begin{gathered}
S=\frac{G_{\mathrm{L}}^{\prime}-G_{\mathrm{M}}^{\prime}}{G_{\mathrm{L}}^{\prime}}, \\
T=\frac{\eta_{\mathrm{L}}^{\prime}-\eta_{\mathrm{M}}^{\prime}}{\eta_{\mathrm{L}}^{\prime}},
\end{gathered}
$$

where $G_{\mathrm{L}}^{\prime}$ and $G_{\mathrm{M}}^{\prime}$ are the large-strain and the minimum-strain elastic moduli, which are defined as the secant modulus at the maximum strain and the tangent modulus at zero instantaneous strain, respectively. Similarly, $\eta_{\mathrm{L}}^{\prime}$ and $\eta_{\mathrm{M}}^{\prime}$ are the instantaneous viscosities at the largest and smallest shear rates, respectively. Then $S>0$ corresponds to strain hardening, $S<0$ to strain softening, and $T>0$ to shear thickening, $T<0$ to shear thinning.

Recently, it has been proposed [Poulos et al. (2013)] to decompose the total stress into an harmonic component $\sigma_{1}(t, \omega)$, corresponding to the first harmonic response provided by the rheometer

$$
\sigma_{1}(t, \omega)=\gamma_{0}\left[G_{1}^{\prime} \sin (\omega t)+G_{1}^{\prime \prime} \cos (\omega t)\right]
$$

and an anharmonic component $\sigma_{\mathrm{anh}}(t, \omega)$, which contains all the higher harmonics contributions

$$
\sigma_{\mathrm{anh}}(t, \omega)=\left|\sigma_{\mathrm{tot}}(t, \omega)\right|-\left|\sigma_{1}(t, \omega)\right| .
$$

This approach is simpler than the FT analysis and the decomposition into Chebyshev polynomials since only the standard output of the rheometer, i.e., the moduli $G_{1}^{\prime}$ and $G_{1}^{\prime \prime}$ in the harmonic approximation, is needed to calculate the anharmonic contribution. Nevertheless, it retains the full information contained in the higher harmonics, in contrast to the approach solely based on the third harmonic [Neidhofer et al. (2003); Ewoldt et al. (2008)]. Moreover, anharmonic moduli which describe the higher harmonic contributions at maximum strain and maximum strain rate, respectively, can be defined by

$$
\begin{aligned}
& G_{\mathrm{anh}}^{\prime}=\frac{\sigma_{\mathrm{anh}}\left(\gamma(t)=\gamma_{0}\right)}{\gamma_{0}}, \\
& G_{\mathrm{anh}}^{\prime \prime}=\frac{\sigma_{\mathrm{anh}}(\gamma(t)=0)}{\gamma_{0}} .
\end{aligned}
$$

Positive $G_{\text {anh }}^{\prime}$ and $G_{\text {anh }}^{\prime \prime}$ correspond to strain hardening and shear thickening, respectively, while negative values correspond to strain softening and shear thinning, respectively. Furthermore, $G_{\text {anh }}^{\prime}$ and $G_{\text {anh }}^{\prime \prime}$ can be related to $G_{\mathrm{L}}^{\prime}$ and $\eta_{\mathrm{L}}^{\prime}$ [Eqs. (3) and (4)] [Poulos et al. (2013)]. The anharmonic moduli, $G_{\text {anh }}^{\prime}$ and $G_{\text {anh }}^{\prime \prime}$, can directly be compared to the ones obtained in the harmonic approximation, $G_{1}^{\prime}$ and $G_{1}^{\prime \prime}$, thus providing a quantitative measure of the anharmonic contributions to the stress response, which is easy to interpret. This comparison also allows one to estimate the extent to which the harmonic approximation describes the full response of the system. 
Finally, it has been proposed to view the LAOS response as a succession of elastic and viscous processes when the strain amplitude exceeds the yield strain [Renou et al. (2010); Rogers et al. (2011)], although a direct relation with steady shear flow curves is not always possible [Poulos et al. (2013)]. In the following, we will not apply this concept to interpret the LAOS response of our colloid-polymer gels but use the FT analysis and the decompositions into Chebyshev polynomials as well as harmonic and anharmonic stresses.

\section{B. LS-Echo}

Echo-DWS or LS-Echo [Hebraud et al. (1997); Höler et al. (1997); Petekidis et al. (2002b)] is based on light multiply scattered by turbid samples. Changes in the time correlation of the scattered light upon application of oscillatory shear are exploited to determine shear-induced particle dynamics, namely, particle rearrangements. Based on the time-dependent scattered intensity, $I(t)$, the normalized time correlation function is calculated as $g^{(2)}(\Delta t)=\left\langle I\left(t_{0}\right) I\left(t_{0}+\Delta t\right)\right\rangle /\langle I(t)\rangle^{2}$, where $\Delta t$ is the delay time and \langle\rangle indicates an average over the start time $t_{0}$. If the system responds elastically, i.e., if the particles return to their position after an integer number of oscillation periods, the speckle pattern changes during the oscillations but returns to the same pattern after $\Delta t=m T$. Therefore, $g^{(2)}(\Delta t)-1$ assumes its maximum value, namely, 1 , at delay times $\Delta t=m T$; it exhibits echoes. In contrast, an echo amplitude below 1 indicates shear-induced irreversible particle rearrangements with the reduction of the amplitude characterizing the degree of irreversibility. Therefore, based on the reversibility or irreversibility of particle motions, Echo-DWS can provide information on an elastic-to-plastic transition.

During the application of an oscillatory shear deformation, particles rearrange due to Brownian and shear-induced motions by $\left\langle\Delta r^{2}(\Delta t)\right\rangle_{\mathrm{B}}$ and $\left\langle\Delta r^{2}(\Delta t)\right\rangle_{\mathrm{S}}$, respectively. Therefore, the correlation function is given by [Petekidis et al. (2002b)]

$$
P(\Delta t)=\sqrt{g^{(2)}(\Delta t)-1} \approx \exp \left[-\frac{1}{6} N k^{2}\left(\left\langle\Delta r^{2}(\Delta t)\right\rangle_{\mathrm{B}}+\left\langle\Delta r^{2}(\Delta t)\right\rangle_{\mathrm{S}}\right)\right],
$$

where $k=2 \pi / \lambda$ with the wavelength $\lambda$ and $N$ is the average number of times a photon is scattered in the sample. Thus, the shear-induced displacements can be separated from the Brownian motion by considering

$$
\frac{P(\Delta t)}{P_{0}(\Delta t)}=\frac{P(\Delta t)}{\lim _{\gamma_{0} \rightarrow 0} P(\Delta t)} \approx \exp \left[-\frac{1}{6} N k^{2}\left\langle\Delta r^{2}(\Delta t)\right\rangle_{\mathrm{S}}\right] .
$$

The nontrivial nonaffine part of the shear-induced motion is obtained at integer multiples of the oscillation time, $\Delta t=m T$, i.e., at the echoes. Hence, the decay of $P(m T) / P_{0}(m T)$ reflects the shear-induced irreversible particle rearrangements.

The length scale probed, $l_{\mathrm{DWS}}$, is controlled by the experimental conditions. If the sample scatters strongly, $l_{\mathrm{DWS}}=\sqrt{6 / N} / k$. However, as in previous work [Petekidis et al. (2002a); Smith et al. (2007); Derakhshandeh et al. (2013)], on average, the photons have been scattered only twice. It has been shown that in the case of on average double-scattered light Eq. (9) still holds [Petekidis et al. (2002a)], and accordingly also the expression of $l_{\text {Dws. }}$. In our experiments $(\lambda=632.8 \mathrm{~nm}$ and $R=137 \mathrm{~nm})$, thus the dynamics on a length scale $l_{\text {DWS }} \approx \sqrt{3} / k \approx R$ are probed. 


\section{MATERIALS AND METHODS}

\section{A. Samples}

The samples are mixtures of polymethylmethacrylate (PMMA) hard-sphere like colloids and linear polystyrene (PS) (Echo-DWS) or polybutadiene (PB) (LAOS) dispersed in cis-decalin (Echo-DWS) or octadecene (LAOS), respectively, at a temperature $T=20^{\circ} \mathrm{C}$. Using dynamic light scattering (DLS), we determined the average hydrodynamic radius of the PMMA particles in cis-decalin, $R^{\mathrm{dec}}=137 \mathrm{~nm}$, and octadecene, $R^{\text {oct }}=154 \mathrm{~nm}$. The samples crystallize neither in quiescent conditions nor under shear, indicating a colloid polydispersity of at least $12 \%$. The radius of gyration of the PS (molecular weight $M_{\mathrm{w}}=132900 \mathrm{~g} / \mathrm{mol}$, from Polymer Laboratories) in cis-decalin was estimated as $r_{\mathrm{g}}^{\mathrm{PS}}=10.8 \mathrm{~nm}$ [Berry (1966)] and the polydispersity was specified by the supplier as $M_{\mathrm{w}} / M_{\mathrm{n}}=1.01$. By DLS, we determined $r_{\mathrm{g}}^{\mathrm{PB}}=12.3 \mathrm{~nm}$ for the PB ( $M_{w}=140000 \mathrm{~g} / \mathrm{mol}$, from Polymer Standard Solutions) in octadecene. This implies a polymer-colloid size ratio in dilute solution $\xi^{\mathrm{PS}}=r_{\mathrm{g}}^{\mathrm{PS}} / R^{\mathrm{dec}}=0.079 \pm 0.013$ for PMMA-PS mixtures and $\xi^{\mathrm{PB}}=r_{\mathrm{g}}^{\mathrm{PB}} / R^{\text {oct }}=0.080 \pm 0.015$ for PMMA-PB mixtures. We calculated the effective polymer-colloid size ratio $\xi^{*}$ for a colloid volume fraction $\phi=$ 0.4 and the two polymer concentrations $c_{\mathrm{p}} / c_{\mathrm{p}}^{*}=0.5$ and 1.0 , where $c_{\mathrm{p}}^{*}=3 M_{\mathrm{w}} / 4 \pi N_{\mathrm{A}} r_{\mathrm{g}}^{3}$ is the overlap concentration and $N_{\mathrm{A}}$ is Avogadro's number, considering the concentration dependence of the polymer size and the mesh size in the semi-dilute regime, according to the generalized free volume theory (GFVT) [Fleer and Tuinier (2007); Lekkerkerker et al. (1992); Aarts et al. (2002)] (Table I). Although our conditions approach those of a $\theta$ solvent, the calculations are based on a good solvent and thus might slightly overestimate the values of $\xi^{*}$. Furthermore, the Asakura-Oosawa (AO) potential strictly does only model the colloid-colloid interactions below $c_{\mathrm{p}}^{*}$ [Fuchs and Schweizer (2002)].

The samples were prepared by mixing appropriate amounts of colloid and polymer stock solutions. A colloid stock solution with volume fraction $\phi=0.6$ was obtained by diluting a sediment, whose volume fraction was estimated to be $\phi=0.67$ based on simulation results [Schaertl and Silescu (1994)]. Polymer stock solutions with two different concentrations were prepared by mixing dry polymer with solvent. The concentration $c_{\mathrm{p}}$ (mass/volume) was calculated from the weighed masses of the two components and the solvent densities. The polymer concentration in the free volume $c_{\mathrm{p}}^{\text {free }}$ was estimated using GFVT [Fleer and Tuinier (2007); Lekkerkerker et al. (1992); Aarts et al. (2002)]. Colloid-polymer mixtures were homogenized using a vortex mixer and then left for at least one day in a rotating-wheel mixer. The samples and their compositions are given in Table I.

TABLE I. Composition of the samples, which are labeled by their nominal polymer concentration. Colloid volume fraction (in the total volume), $\phi$, polymer concentrations in the total, $c_{\mathrm{p}} / c_{\mathrm{p}}^{*}$, and free, $c_{\mathrm{p}}^{\text {free }} / c_{\mathrm{p}}^{*}$, volume normalized by the overlap concentration, $c_{\mathrm{p}}^{*}$, effective polymer-colloid size ratio, $\xi^{*}$, and depth of the attractive potential $U_{0}$ normalized by the thermal energy $k_{\mathrm{B}} T$.

\begin{tabular}{lccccc}
\hline \hline$c_{\mathrm{p}} / c_{\mathrm{p}}^{*}$ (nominal) & $\phi$ & $c_{\mathrm{p}} / c_{\mathrm{p}}^{*}$ & $c_{\mathrm{p}}^{\text {free }} / c_{\mathrm{p}}^{*}$ & $\xi^{*}$ & $U_{0} / k_{\mathrm{B}} T$ \\
\hline PMMA-PS decalin & & & & & \\
0.5 & 0.41 & 0.48 & 0.90 & $0.048 \pm 0.006$ & -23.8 \\
1 & 0.40 & 0.99 & 1.78 & $0.033 \pm 0.005$ & -48.0 \\
PMMA-PB octadecene & & & & & -24.2 \\
0.5 & 0.40 & 0.49 & 0.92 & $0.050 \pm 0.007$ & -46.5 \\
1 & 0.39 & 0.98 & 1.75 & $0.034 \pm 0.007$ & \\
\hline \hline
\end{tabular}




\section{B. Rheology}

Rheological measurements were performed using an MCR 501 stress-controlled rheometer with cone and plate geometries of diameter $40 \mathrm{~mm}$, cone angle $2^{\circ}$, and gap size $0.054 \mathrm{~mm}$. In order to minimize wall slip, which typically strongly affects the rheological response of colloid-polymer gels [Ballesta et al. (2013)], we used geometries with a roughness of the order of several particle diameters [Ballesta et al. (2013)]. Using these roughened geometries, no wall-slip effects were observed, in agreement with previous results [Laurati et al. (2011)]. The temperature was set to $T=20^{\circ} \mathrm{C}$ and controlled within $\pm 0.1{ }^{\circ} \mathrm{C}$ using a standard Peltier plate. The effects of sample loading and aging were reduced by performing the following rejuvenation procedure before each test. Directly after loading, a dynamic strain sweep (DSS) was performed to estimate the strain amplitude $\gamma_{0}$ at which the system starts to flow, i.e., oscillatory shear was applied to the samples with frequency $\omega=0.1 \mathrm{rad} / \mathrm{s}$ and increasing $\gamma_{0}$ until the sample showed a liquidlike response. Then, before each measurement, flow of the sample was induced by applying oscillatory shear at a sufficiently large strain amplitude; $\gamma_{0}=1000 \%$ was large enough to induce flow in both samples. Shear was applied until a steady-state response, i.e., time-independent storage, $G^{\prime}$, and loss, $G^{\prime \prime}$, moduli, were observed, which typically took about 300 s. Subsequently, oscillatory shear at the same frequency, but in the linear viscoelastic regime at $\gamma_{0}=0.1 \%$, was applied (and the recovery of the sample elasticity monitored) until the linear viscoelastic moduli reached a steady-state value, typically after 500-1000 s (depending on sample). This indicates that no further structural changes occurred and hence a reproducible state of the sample was reached before a new measurement was started. Note that ageing effects might be present at longer waiting times.

Dynamic frequency sweeps (DFSs) in the linear viscoelastic regime at $\gamma_{0}=0.1 \%$, DSSs at $\omega=0.5$ and $1.0 \mathrm{rad} / \mathrm{s}$ and, for the LAOS analysis, a series of dynamic time sweeps (DTSs) at different strain amplitudes and $\omega=0.5$ and $1.0 \mathrm{rad} / \mathrm{s}$ were measured. In DTS measurements, $\gamma_{0}$ was varied between $0.1 \%$ and $600 \%$. Once a stationary LAOS response was reached, the last full waveform of stress and strain was recorded and stored. The direct strain oscillation option of the Physica MCR 501 was used to ensure a perfect sinusoidal strain at all amplitudes. The possibility to perform LAOS experiments with a stress-controlled rheometer has recently been demonstrated for a variety of different samples [Laeueger and Steetin (2010)].

\section{LS-Echo}

During the LS-Echo measurements, shear was applied by means of a home-built shear cell with two parallel glass plates [Petekidis et al. (2002b)], which were separated by about $1 \mathrm{~mm}$. The top plate is pushed by a piezoelectric actuator which is driven by a sinusoidal signal $\gamma(t)=\gamma_{0} \sin (\omega t)$ of frequency $\omega=2 \pi / T$ and provides a maximum displacement of about $90 \mathrm{~nm}$. The top plate is connected to a lever which transmits an enhanced displacement to the bottom plate, which moves in the opposite direction than the top plate. With this arrangement, maximum strain amplitudes $\gamma_{0} \sim 100 \%$ can be achieved.

The sample is illuminated from below by a helium-neon laser (wavelength $\lambda=632.8 \mathrm{~nm}$ ), and the scattered light is detected above the sample by a single-mode fiber connected to an avalanche photodiode. A polarizer perpendicular to the laser polarization is added in front of the fiber to remove any (polarized) single-scattered light. The signal is processed by a Flex410R digital correlator from Correlator.com to obtain the normalized time correlation function $g^{(2)}(\Delta t)$. Additional details on the setup are given in the work of Petekidis et al. (2002b). 
Glasses and gels are characterized by nonergodic dynamics. In an Echo-DWS or LSEcho experiment, several speckles are probed during one period of oscillation: The width of the echo peak corresponds approximately to the time needed for a speckle to cross the detector [Pham et al. (2004)]. For the strain amplitudes probed in our experiments $\left(1 \% \leq \gamma_{0} \leq 18 \%\right)$, this width varies from $\mathrm{T} / 100$ to approximately $\mathrm{T} / 2000$, with $\mathrm{T}$ the oscillation period. This implies that of the order of 100-2000 speckles are sampled. Moreover, the laser beam is relatively large (typically, the beam cross section is at least 20 times larger than in a DLS experiment, as the beam is not focused) and hence a larger volume of the sample is probed. In addition, the speckles are smaller, also due to the multiple scattering events. The degree of remaining nonergodicity is indicated by the fluctuations of the intercept of the correlation function $\left[g^{(2)}(\Delta t=0)\right.$, not shown] which at low strain amplitudes is of the order of $10 \%-20 \%$ but rapidly decreases as the strain amplitude increases. According to these arguments, a reasonable ensemble average is performed during our LS-Echo measurements.

\section{RESULTS AND DISCUSSION}

\section{A. Linear viscoelasticity}

The linear viscoelastic moduli $G^{\prime}(\omega)$ and $G^{\prime \prime}(\omega)$, measured in DFSs, indicate $G^{\prime}>G^{\prime \prime}$, i.e., a solidlike response, within the experimental frequency window, as shown for PMMA-PB mixtures in Fig. 1, and for PMMA-PS mixtures in previous work [Laurati et al. (2009)]. This indicates that both samples are gels, in agreement with the previously determined gelation boundary at $c_{\mathrm{p}} / c^{*}=0.4$ [Laurati et al. (2009)]. For the PMMA-PB and PMMA-PS mixtures, the storage moduli $G^{\prime}$ at $\omega=1 \mathrm{rad} / \mathrm{s}$, normalized by $k_{B} T / R^{3}$ to take into account the different energy densities, are shown for an extended range of $c_{p} / c_{p}^{*}$ in the inset of Fig. 1. The values for the PMMA-PB and PMMA-PS mixtures are comparable and show a similar dependence on $c_{p} / c_{p}^{*}$. For the PMMA-PB mixtures, $G^{\prime}$ in the gel

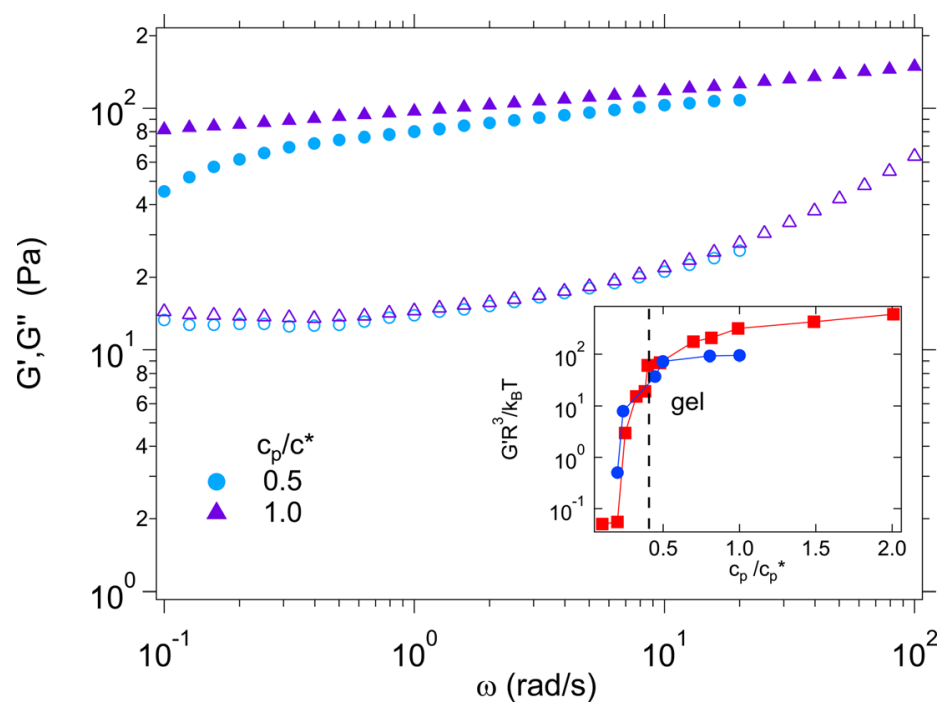

FIG. 1. Storage modulus $G^{\prime}(\omega)$ (full symbols) and loss modulus $G^{\prime \prime}(\omega)$ (open symbols) as a function of frequency $\omega$ in the linear viscoelastic regime for polymer concentrations $c_{\mathrm{p}} / c^{*}=0.5(\bullet)$ and $c_{\mathrm{p}} / c^{*}=1.0(\mathbf{\Delta})$ of PMMA-PB mixtures. Inset: $G^{\prime}(\omega=1 \mathrm{rad} / \mathrm{s}) R^{3} / k_{B} T$ as a function of $c_{\mathrm{p}} / c^{*}$, for PMMA-PS mixtures in decalin (घ) and PMMA-PB mixtures in octadecene $(\bullet)$. 
state is smaller by a factor of $2-3$, which could be due to small differences in volume fraction and/or larger softness of the colloids in octadecene, as suggested by their slightly larger radius: $R^{\text {oct }}>R^{\text {dec }}$. The gel with $c_{p} / c^{*}=1.0$ shows a stronger elastic response than the gel with $c_{p} / c^{*}=0.5$. This is due to the stronger intercluster bonds, which are responsible for the network structure of the gels and hence their viscoelastic response [Laurati et al. (2009); Zaccone and Del Gado (2009)].

\section{B. DSSs}

Based on DSS measurements (Fig. 2), the yielding behavior is investigated on an average level, i.e., approximating the stress response by the first harmonic contribution only. For both samples, a first yield point, taken as the maximum of $G^{\prime \prime}\left(\gamma_{0}\right)$, is observed at a strain amplitude $\gamma_{0} \approx 1 \%$ and has been associated with the breaking of the first intercluster bonds [Laurati et al. (2011); Koumakis and Petekidis (2011)]. At slightly larger strains, about $2-3 \%, G^{\prime}\left(\gamma_{0}\right)$ and $G^{\prime \prime}\left(\gamma_{0}\right)$ cross, which indicates the onset of a fluidlike response and has been related to the failure of the network structure [Laurati et al.

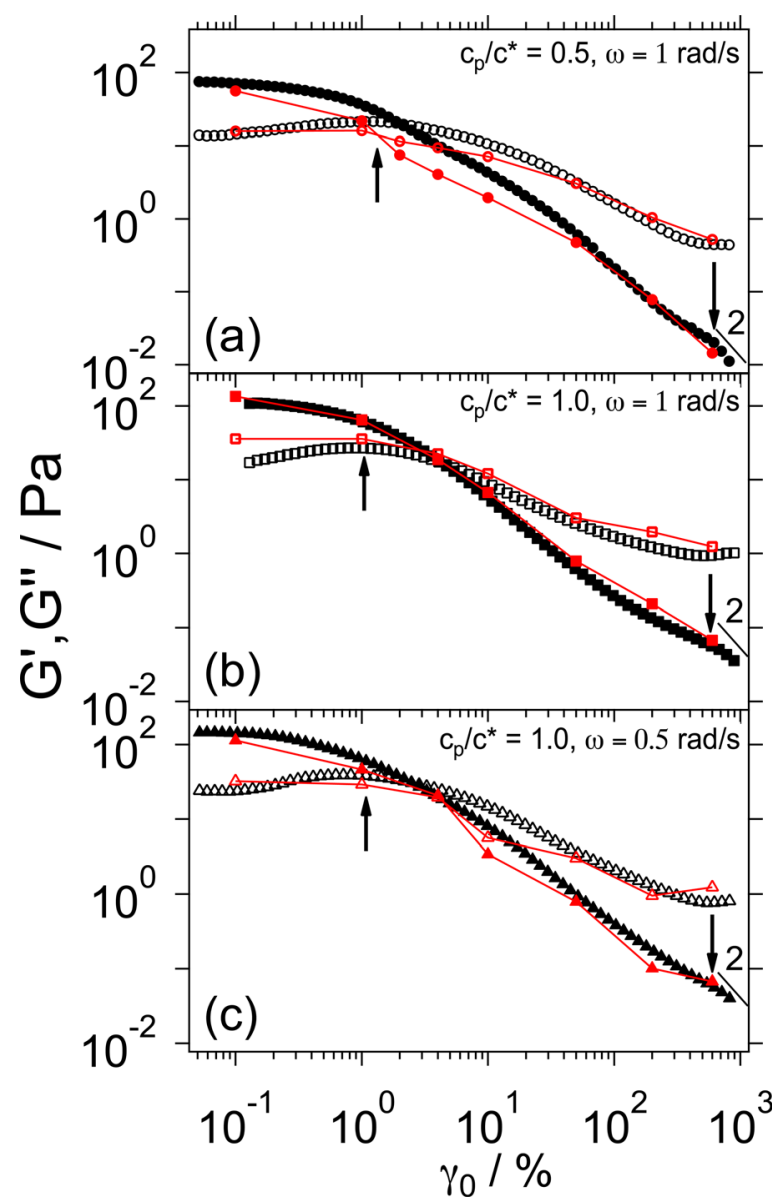

FIG. 2. Storage modulus $G^{\prime}$ (full symbols) and loss modulus $G^{\prime \prime}$ (open symbols) as a function of strain amplitude $\gamma_{0}$ for (a) $c_{\mathrm{p}} / c^{*}=0.5$ and $\omega=1 \mathrm{rad} / \mathrm{s}$, (b) $c_{\mathrm{p}} / c^{*}=1.0$ and $\omega=1 \mathrm{rad} / \mathrm{s}$, and (c) $c_{\mathrm{p}} / c^{*}=1.0$ and $\omega=0.5 \mathrm{rad} / \mathrm{s}$ for PMMA-PB mixtures. Black symbols represent results of DSS measurements, red lines and symbols steady-state values obtained in DTS measurements. Arrows indicate the yield points and the short lines slopes of 2 . 
(2011); Koumakis and Petekidis (2011)]. For larger strains, $\gamma_{0} \gtrsim 3 \%$, the response is complex but does not show the response expected for shear thinning concentrated colloids, namely, $G^{\prime} \sim \gamma_{0}^{-\nu^{\prime}}$ and $G^{\prime \prime} \sim \gamma_{0}^{-\nu^{\prime \prime}}$ with $0.6<\nu^{\prime \prime}<1.0$ and $\nu^{\prime} \approx 2 \nu^{\prime \prime}$ [Brader et al. (2010); Koumakis et al. (2012)], although a different ratio of the exponents, $\nu^{\prime} \approx 1.5 \nu^{\prime \prime}$, has been observed in different systems, in certain ranges of $\omega$ and $\phi$ [Koumakis et al. (2012); Poulos et al. (2013)]. Only at very large strain amplitudes $\gamma_{0}>500 \%$, where an inflection of $G^{\prime}\left(\gamma_{0}\right)$ is seen, the data indicate such a response. Such response has previously been attributed to the presence of a second yield point beyond which most of the colloidal network and clusters are disaggregated [Laurati et al. (2011); Koumakis and Petekidis (2011)]. Two yield points are also observed in attractive colloidal glasses [Pham et al. (2006); Pham et al. (2008)], where one indicates bond breaking, as in the present case, and the other, in contrast to our system, cage breaking which is associated with the onset of shear thinning.

Measurements performed at different frequencies $\omega$ [for $c_{\mathrm{p}} / c^{*}=1.0$, Figs. 2(b) and 2(c)] indicate that with increasing $\omega$ the first yield strain and in particular the crossing point of $G^{\prime}$ and $G^{\prime \prime}$ slightly increase. This trend agrees with previous findings, where it was attributed to the reduced opportunity for bond breaking in a shorter cycle, i.e., at higher $\omega$ [Laurati et al. (2011)].

\section{LAOS}

In order to investigate the in-cycle response and the higher order harmonic contributions to the stress response, we performed DTS measurements at different strain amplitudes. Once the response reached a steady state, during one cycle the time-dependent stress signal was extracted (Sec. III B) and analyzed using different methods. The storage, $G^{\prime}$, and loss, $G^{\prime \prime}$, moduli obtained by the first harmonic approximation are in qualitative agreement with the results of DSS measurements (Fig. 2), which correspond to DTS data at short times. Only for the sample with $c_{\mathrm{p}} / c^{*}=0.5$ and $\omega=1 \mathrm{rad} / \mathrm{s}$, at intermediate $\gamma_{0}$ the moduli determined by DSS are slightly larger than those measured in DTS.

\section{Lissajous figures}

The onset of a nonlinear stress response to oscillatory shear can be visualized by Lissajous figures, in which the stress $\sigma$ is shown as a function of either strain $\gamma$ or shear rate $\dot{\gamma}$ to highlight the elastic and viscous parts of the response, respectively (Fig. 3). If the stress response is linear, the Lissajous figures are elliptical, while any deviation from this shape indicates the presence of higher harmonic components. For both samples, the elastic and viscous Lissajous figures for a strain $\gamma_{0}=0.1 \%$ are elliptical, indicating a linear response of the system. At $\gamma_{0}=1 \%$, the shape of the Lissajous figures slightly deviates from an ellipse, i.e., the response already is nonlinear. In the elastic figures, at maximum strain (zero rate), the deformation is reversed and the stress initially increases almost linearly with $\gamma$ until the in-cycle yielding takes place and the system starts to flow at almost constant stress. At this strain, the in-cycle yield strain almost coincides with the maximum strain amplitude and the flow regime only extends over a very short fraction of the cycle. Increasing $\gamma_{0}$ the flow regime extends over a larger fraction of the cycle, as indicated by the increasingly rectangular shape of the elastic Lissajous figures. Similarly, in the viscous figures, an increasingly larger deviation from the elliptical shape and an increasingly extended part of almost constant stress approaching the maximum in-cycle rate, indicate that shear thinning becomes more prominent at larger strain amplitudes. 

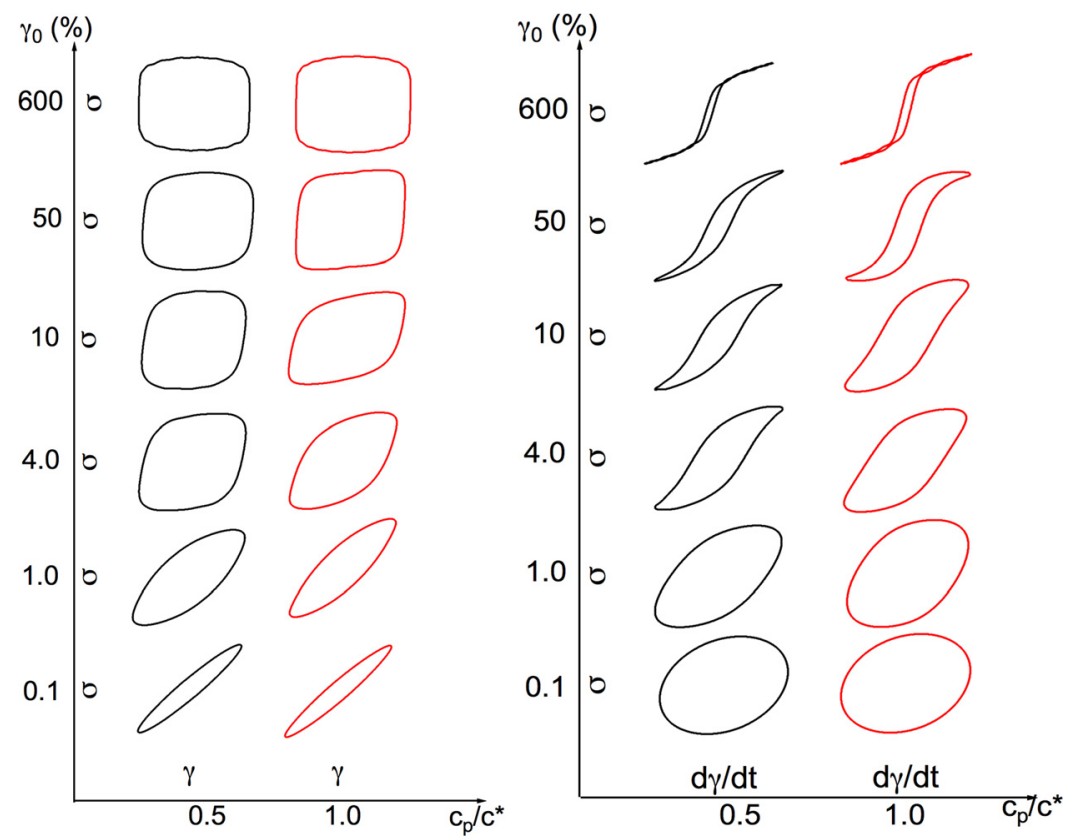

FIG. 3. Elastic (left) and viscous (right) Lissajous figures showing the stress $\sigma$ as a function of strain $\gamma$ and shear rate $\dot{\gamma}$, respectively, for PMMA-PB samples with polymer concentrations $c_{\mathrm{p}} / c^{*}=0.5$ and 1.0 , as indicated on the horizontal axes. The strain amplitude $\gamma_{0}$ is indicated on the vertical axes. Frequency $\omega=1 \mathrm{rad} / \mathrm{s}$.

\section{FT analysis}

The time-dependent stress response was Fourier transformed to extract the amplitudes of all harmonic contributions. Even harmonic contributions are absent, indicating that the measurements are not affected by wall slip or shear banding. All odd higher harmonics show similar dependencies on the strain amplitude $\gamma_{0}$ and frequency $\omega$ with the amplitudes of the third harmonics considerably exceeding the other higher harmonics. Hence, we concentrate on the amplitudes of the third harmonics, $I_{3}$, compared to the ones of the first harmonics, $I_{1}$. The $\gamma_{0}$ dependence of the ratio $I_{3} / I_{1}$ shows that, for all samples, already at $\gamma_{0}=1 \%$ the third harmonics become significant (Fig. 4) and hence the stress response nonlinear, in agreement with the results of the DSS measurements (Fig. 2). With increasing $\gamma_{0}$, the third harmonic contributions increase and then saturate or decrease at large strains $\left(\gamma_{0}>50 \%\right.$ for $c_{\mathrm{p}} / c^{*}=1.0$, and $\gamma_{0}>200 \%$ for $\left.c_{\mathrm{p}} / c^{*}=0.5\right)$. The increase reflects an increasingly pronounced plastic response with the stronger gel, i.e., $c_{\mathrm{p}} / c^{*}=1.0$, showing a maximum value $I_{3} / I_{1} \approx 32 \%$. This is close to $33 \%$, the value corresponding to a perfectly plastic response, where the stress follows a square wave [Wilhelm et al. (2000); Ewoldt et al. (2010)]. The growth of the nonlinear contributions with increasing $\gamma_{0}$ is less abrupt than in hard-sphere, star polymer, and starlike micelle glasses, [Koumakis et al. (2012); Renou et al. (2010); Poulos et al. (2013)] suggesting a more gradual yield process that extends over a wide range of strain amplitudes. The decrease of $I_{3} / I_{1}$ at large $\gamma_{0}$ is attributed to the approach toward a simpler liquidlike response, as also observed in hard-sphere glasses [Koumakis et al. (2012)], concentrated microgel dispersions [Koumakis et al. (2012); Le Grand and Petekidis (2008); Carrier and Petekidis (2009)], multiarm star polymers [Rogers et al. (2011)], and starlike micelles [Poulos et al. (2013)]. For the sample with $c_{\mathrm{p}} / c^{*}=1.0, I_{3} / I_{1}$ is larger at smaller frequencies $\omega$ for the same strain value, which corresponds to the observation that the 


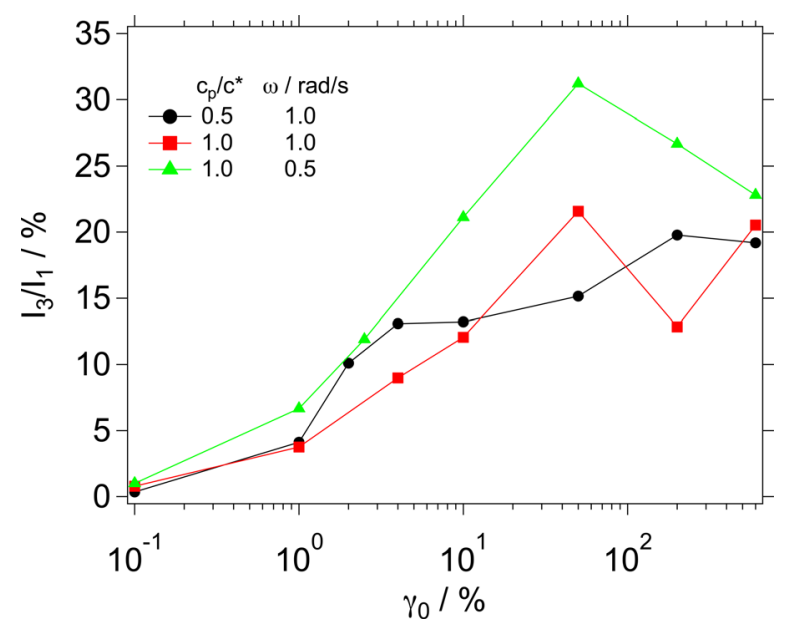

FIG. 4. FT analysis results for PMMA-PB samples with polymer concentrations $c_{\mathrm{p}} / c^{*}=0.5$ and 1.0 , and frequencies $\omega=0.5$ and $1.0 \mathrm{rad} / \mathrm{s}$, as indicated, showing the amplitude of the third harmonic divided by that of the first, $I_{3} / I_{1}$, as a function of strain amplitude $\gamma_{0}$.

yield strain decreases with decreasing $\omega$ [Figs. 2(b) and 2(c)], and therefore, at smaller $\omega$, the nonlinear regime occurs earlier.

\section{Stress expansion using Chebyshev polynomials}

Based on the raw stress signal during the DTS measurements, we extracted the third order elastic, $e_{3}$, and viscous, $v_{3}$, coefficients [Eqs. (1) and (2)], and the related $S$ and $T$ nonlinearity indexes [Eqs. (3) and (4)]. For both samples, starting at $\gamma_{0}=1 \%$, they show a positive and increasing elastic contribution and an essentially negative and decreasing viscous contribution [Figs. 5(a) and 5(b)]. This indicates that, with increasing deformation, the sample is strain hardening and shear thinning, as already suggested by the Lissajous figures. However, at $\gamma_{0}=1 \%$ elastic and viscous coefficients are both positive, which implies that the sample is strain hardening and shear thickening. At $\gamma_{0} \approx 1 \%$, the first yield strain was observed in the DSS measurements (Fig. 2), which was previously associated with the breaking of the first intercluster bonds [Laurati et al. (2011); Koumakis and Petekidis (2011)] and thus the onset of network disruption. The breaking of bonds implies an increased energy dissipation and hence a higher viscosity, which appears as shear thickening. In addition, the breaking of bonds is expected to lead to a reduced elastic response and therefore strain softening. However, not all bonds are broken at this $\gamma_{0}$, but, according to previous work, mainly intercluster bonds break [Laurati et al. (2011); Zaccone and Del Gado (2009)]. It is conceivable that as intercluster bonds break, stronger network or cluster structures form induced by the strain deformation, which results in average strain hardening.

The relative contribution of the elastic and viscous in-cycle responses can be estimated by comparing absolute values of the elastic, $e_{3}$, and viscous, $v_{3}$, Chebyshev coefficients [Fig. 5(c)]. Initially, shear thickening dominates. Then, between the first yield point and the strain amplitude at which $G^{\prime}$ and $G^{\prime \prime}$ cross in DSS measurements, the sample is mainly strain hardening. As previously discussed, the strain hardening could result from a restructuring of the gel structure leading to stronger gel networks or clusters, before it finally yields and starts to flow. At even larger strains, shear thinning starts to dominate and is associated with the fluidization of the sample. 

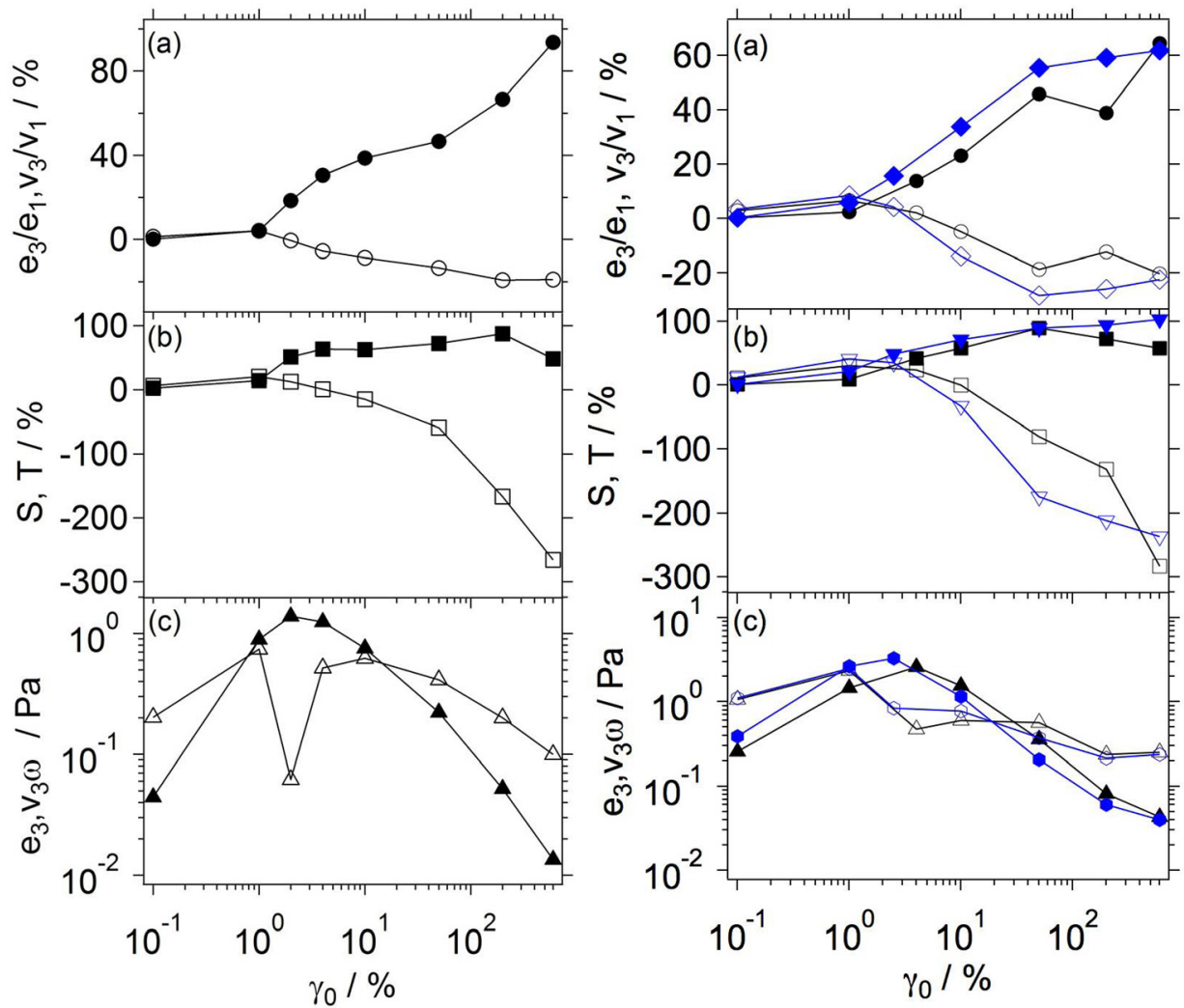

FIG. 5. (a) Relative third order elastic, $e_{3} / e_{1}$ (full symbols) and viscous, $v_{3} / v_{1}$ (open symbols) Chebyshev coefficients, (b) dimensionless indexes of nonlinearity, $S$ (full symbols) and $T$ (open symbols) [Eqs. (3) and (4)], and (c) absolute values of the strain hardening/softening, $e_{3}$ (full symbols), and shear thickening/thinning, $v_{3} \omega$ (open symbols), coefficients, as a function of strain amplitude $\gamma_{0}$, for PMMA-PB samples with (left) polymer concentration $c_{\mathrm{p}} / c^{*}=0.5$ and frequency $\omega=1 \mathrm{rad} / \mathrm{s}$ and (right) $c_{\mathrm{p}} / c^{*}=1.0$ and $\omega=0.5 \mathrm{rad} / \mathrm{s}$ (blue symbols) and 1.0 $\mathrm{rad} / \mathrm{s}$ (black symbols).

For $c_{\mathrm{p}} / c^{*}=0.5$ less pronounced strain hardening and shear thinning behavior are observed at lower frequencies, corresponding to the less pronounced nonlinearities observed in the FT analysis.

\section{Anharmonic moduli}

The anharmonic storage, $G_{\text {anh }}^{\prime}$, and loss, $G_{\text {anh }}^{\prime \prime}$, moduli were calculated according to Eqs. (6)-(8). Comparison to the harmonic storage, $G^{\prime}$, and loss, $G^{\prime \prime}$, moduli reveals that the anharmonic component, i.e., nonlinear contributions, becomes important for $\gamma_{0}>$ $0.1 \%$ (Fig. 6), in agreement with the FT analysis and the Chebyshev polynomials decomposition. For both samples and all values of $\gamma_{0}$, the anharmonic storage modulus $G_{\text {anh }}^{\prime}$ is positive, which indicates in-cycle strain hardening. The magnitude of $G_{\text {anh }}^{\prime}$ increases upto the strain amplitude corresponding to, approximately, the crossing of $G^{\prime}$ and $G^{\prime \prime}$ in the DSS measurements (Fig. 2) and decreases monotonically for larger $\gamma_{0}$. Concomitantly, for both samples, the magnitude of the anharmonic loss modulus, $G_{\text {anh }}^{\prime \prime}$, decreases monotonically. It is positive while $G_{\text {anh }}^{\prime}$ increases and then, after $G_{\text {anh }}^{\prime}$ attained its maximum value, becomes negative while $G_{\text {anh }}^{\prime}$ decreases. This indicates that the process of network and cluster deformation which leads to fluidization is characterized by an initial regime 


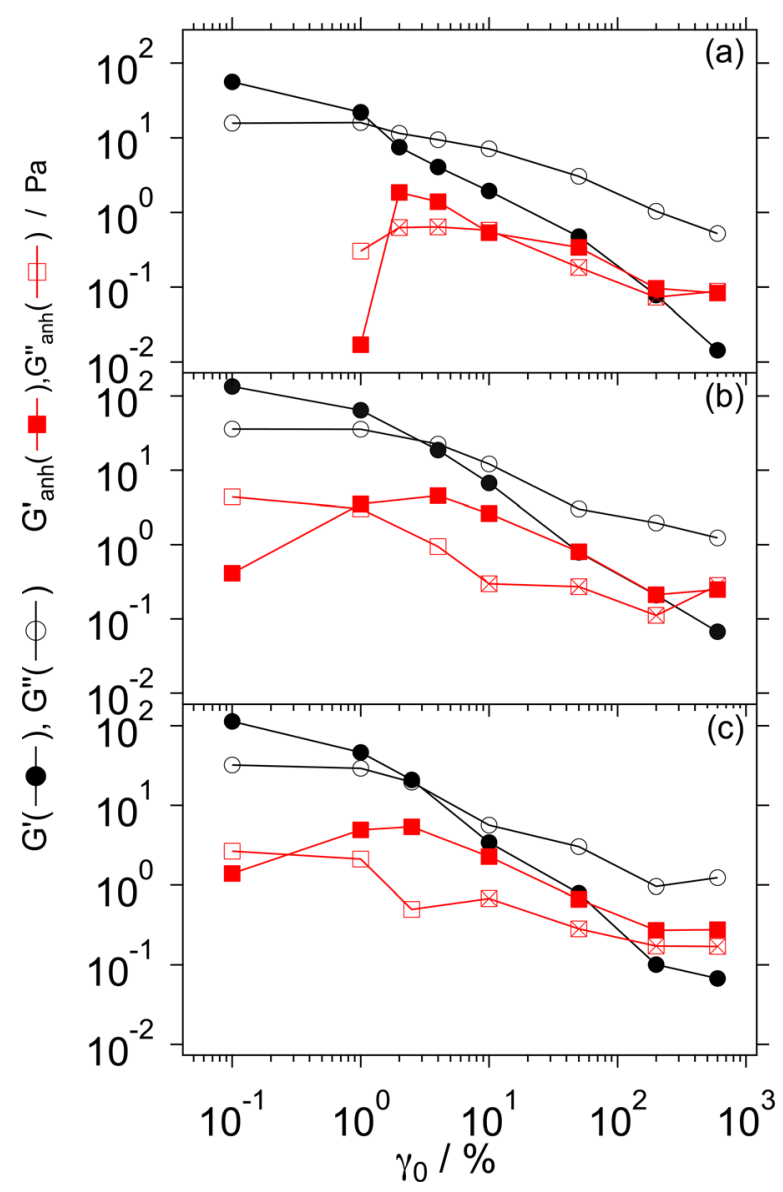

FIG. 6. Storage, $G^{\prime}$, and loss, $G^{\prime \prime}$, modulus in the first harmonic approximation, and anharmonic storage, $G_{\text {anh }}^{\prime}$, and loss, $G_{a n h}^{\prime \prime}$, modulus as a function of strain amplitude $\gamma_{0}$ for PMMA-PB samples with (a) polymer concentration $c_{\mathrm{p}} / c^{*}=0.5$ and frequency $\omega=1 \mathrm{rad} / \mathrm{s}$, (b) $c_{\mathrm{p}} / c^{*}=1.0$ and $\omega=1.0 \mathrm{rad} / \mathrm{s}$, and (c) $c_{\mathrm{p}} / c^{*}=1.0$ and $\omega=0.5 \mathrm{rad} / \mathrm{s}$. For $G_{\text {anh }}^{\prime \prime}$ open symbols indicate positive values, crossed symbols negative values.

where shear thickening and strain hardening coincide. In the discussion of the Chebyshev polynomials analysis, it was suggested that in this regime shear thickening is caused by the increased energy dissipation due to bond breaking, while strain hardening results from the formation of stronger structures during the fragmentation of the network. For $\gamma_{0}$ larger than the crossing point of $G^{\prime}$ and $G^{\prime \prime}$ in harmonic approximation, the anharmonic contributions gradually decrease, indicating that the response of the system increasingly resembles the one of a flowing fluid, consistent with the observation of in-cycle shear thinning. Nevertheless, compared to the Chebyshev polynomials decomposition, some differences are observed. First, the range of $\gamma_{0}$ where shear thickening and strain hardening are simultaneously observed extends to larger $\gamma_{0}$. It coincides with the regime of network restructuring and occurs before the system flows. Second, the anharmonic moduli show that the contribution of strain hardening dominates over shear thinning for essentially all values of $\gamma_{0}\left(\left|G_{\text {anh }}^{\prime}\right|>\left|G_{\text {anh }}^{\prime \prime}\right|\right)$, while in the Chebyshev analysis shear thinning dominates at strain amplitudes $\gamma_{0} \gtrsim 10 \%$. These differences are due to retaining all nonlinear contributions to the stress when calculating the anharmonic moduli, while only the third harmonic nonlinear contribution is considered in the analysis based on the 
Chebyshev polynomials decomposition. This indicates the importance of the higher harmonic contributions.

No clear frequency dependence of the anharmonic contributions can be discerned for $c_{\mathrm{p}} / c^{*}=1.0$ in this analysis.

\section{Summary}

We used different analysis methods to investigate the stress response of the system to LAOS. Lissajous figures provide a qualitative interpretation of the data. They reveal the onset of nonlinear contributions to the stress response at strain amplitudes $\gamma_{0} \geq 1 \%$, which agrees with the other analysis methods. The deviations from linear response can be quantified using the FT analysis. The nonlinear contributions gradually increase up to strain amplitudes of the order of $100 \%$, together with an increasingly plastic response, but start to decrease for $\gamma_{0} \gtrsim 100 \%$, when the response approaches that of a fluid.

Lissajous figures also indicate the presence of shear thickening at $\gamma_{0}=1 \%$ and shear thinning for larger $\gamma_{0}$. The initial shear thickening is attributed to the breaking of intercluster bonds, i.e., the destruction of the network, and the associated energy dissipation. At larger strain amplitudes, namely, once the network is broken, shear thinning occurs and finally the system is fluidized. The analyses based on the decomposition into Chebyshev polynomials and on the anharmonic moduli can be applied to quantify and analyze more in detail the qualitative trends observed in the Lissajous figures and determine the dominant in-cycle responses at different strain amplitudes. While both confirm the findings of the Lissajous figures analysis in terms of shear thinning, they also reveal the presence of strain hardening at all $\gamma_{0}$ where nonlinear contributions are important. Strain hardening could be associated to the formation of stronger network or cluster structures during the process of network destruction. The Chebyshev coefficient analysis seems particularly precise in locating the onset of the nonlinear response. Furthermore, it suggests that shear thinning already sets in during network restructuring, i.e., at strain amplitudes below the crossing of $G^{\prime}$ and $G^{\prime \prime}$ in the harmonic approximation. In contrast, the anharmonic moduli analysis indicates that shear thinning is only observed once the network structure is completely disrupted, i.e., when $G^{\prime \prime}>G^{\prime}$ in the harmonic approximation. Moreover, at strain amplitudes $\gamma_{0}$ where the network structure is disrupted, the dominant in-cycle response is shear thinning according to the Chebyshev coefficient analysis, but strain hardening according to the anharmonic moduli. One expects that deep in the nonlinear regime, i.e., for strain amplitudes significantly larger than $1 \%$, the Chebyshev coefficients analysis, which only considers third harmonic nonlinear contributions, is less accurate than the anharmonic moduli analysis, which retains all high harmonic contributions (including their signs), which become comparable to the third harmonic.

\section{LS-Echo}

LS-Echo measurements were performed while applying oscillatory shear with the cell described in Sec. III C. Larger oscillation frequencies, which are not accessible in the rheometer, can be applied with this shear cell. At the high oscillation frequencies accessible with the shear cell, the effects of wall slip are expected to be small [Ballesta et al. (2013)]. This is particularly important for LS-Echo measurements, in which glass plates are required while geometries with large surface roughness cannot be used, rendering the measurements prone to wall slip.

The effect of wall slip has been tested by recording correlation functions $g^{(2)}(\Delta t)-1$ for different values of the strain amplitude $\gamma_{0}$ and oscillation frequencies $f=\omega / 2 \pi=$ $11 \mathrm{~Hz}$ and $70 \mathrm{~Hz}$ (Fig. 7) and determining the inverse width of the initial decay, $W$, as a 


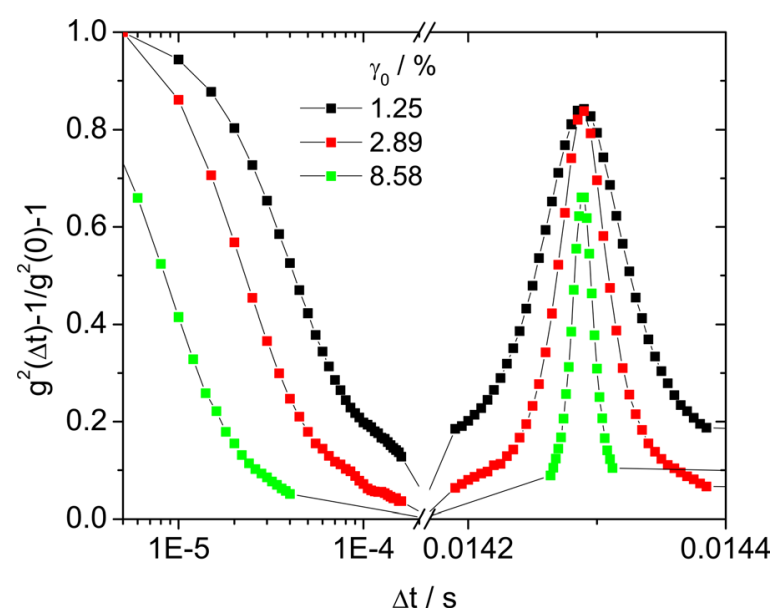

FIG. 7. Correlation functions $g^{(2)}(\Delta t)-1 / g^{(2)}(0)-1$ as a function of delay time $\Delta t$ measured for the PMMAPS sample with polymer concentration $c_{\mathrm{p}} / c^{*}=0.5$ under application of oscillatory shear of frequency $f=70 \mathrm{~Hz}$. Initial decays and first echoes are shown for three different strain amplitudes $\gamma_{0}=1.25 \%, 2.89 \%$, and $8.58 \%$, as indicated.

function of $\gamma_{0}$ (Fig. 8). In the absence of wall slip and/or heterogeneous flow, $W$ is directly proportional to the inverse of the strain rate $1 / \dot{\gamma}$ and therefore a linear dependence of $W$ on $\gamma_{0}$ is expected [Hebraud et al. (1997); Petekidis et al. (2002a)]. We find that for $f=11 \mathrm{~Hz}$ indeed a linear dependence is found for both samples. For $f=70 \mathrm{~Hz}$, similar results are observed for $\gamma_{0}<13 \%$ while $W$ starts to decrease for larger values of $\gamma_{0}$ indicating that slip might affect measurements with $\gamma_{0} \gtrsim 13 \%$ and $f=70 \mathrm{~Hz}$.

Echo correlation functions $g^{(2)}(m T)-1$ (Fig. 9), normalized to their intercepts $g^{(2)}(0)$ -1 , were extracted from $g^{(2)}(\Delta t)-1$ functions by collecting the echo peak values at times $\Delta t=m T$. All $g^{(2)}(m T)-1$ show an initial decay, which is related to the short-time Brownian dynamics, and a decay at long times, which is associated to shear-induced plastic rearrangements of the gel structure or, equivalently, shear-induced $\alpha$-relaxation. With

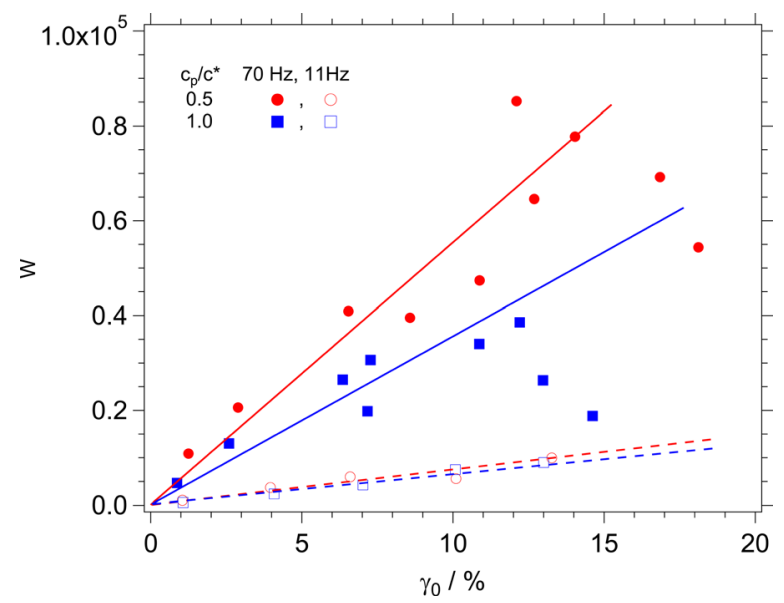

FIG. 8. Inverse width at half height, $W$, of the first echo of the correlation function as a function of strain amplitude $\gamma_{0}$, for PMMA-PS samples with polymer concentrations $c_{\mathrm{p}} / c^{*}=0.5$ and 1.0 and frequencies $f=11$ and $70 \mathrm{~Hz}$, as indicated. 


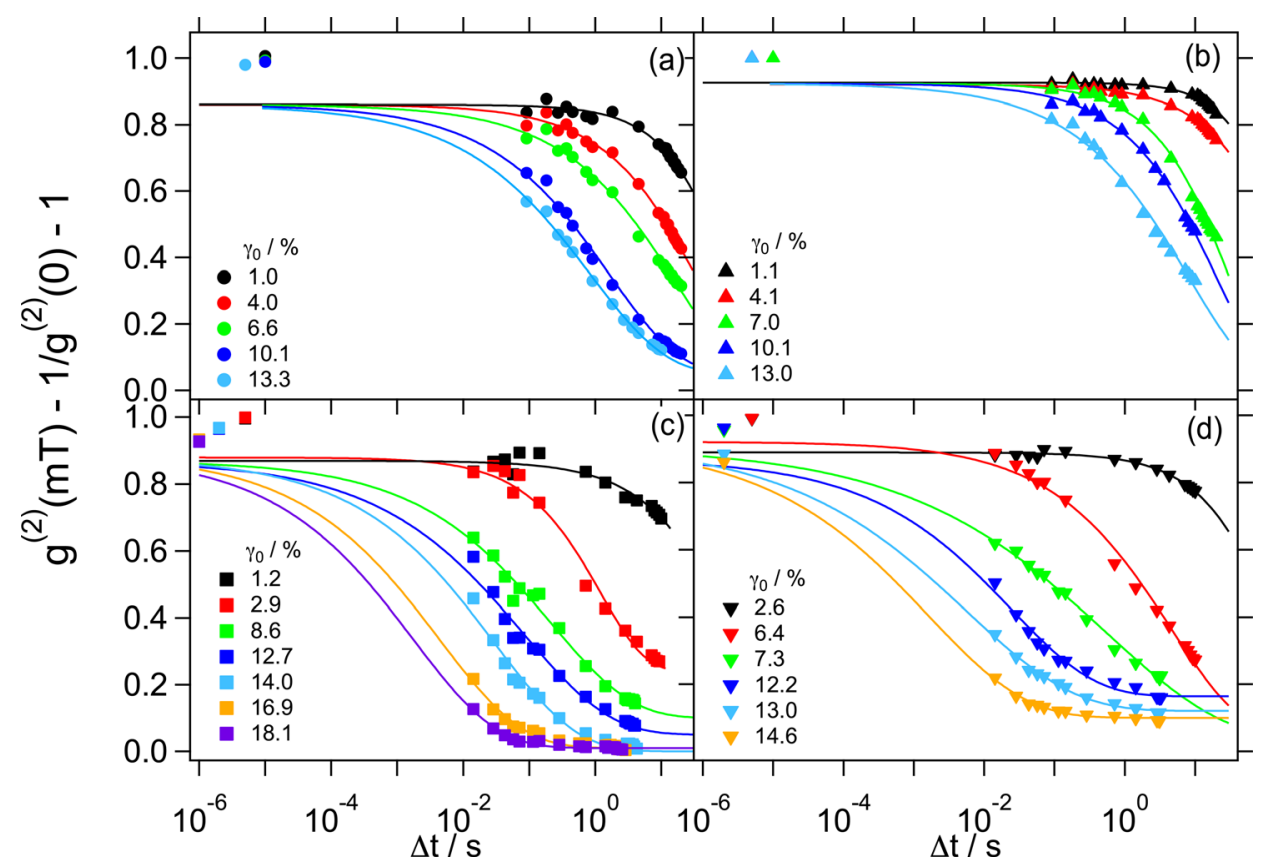

FIG. 9. Correlation functions obtained by Echo-DWS, $g^{(2)}(m T)-1 / g^{(2)}(0)-1$, for PMMA-PS samples with polymer concentrations $c_{\mathrm{p}} / c^{*}=0.5[(\mathrm{a})$ and $(\mathrm{c})]$; and $c_{\mathrm{p}} / c^{*}=1.0[(\mathrm{~b})$ and (d)]; and frequencies $f=11 \mathrm{~Hz}[(\mathrm{a})$ and (b)], and $70 \mathrm{~Hz}$ [(c) and (d)]. Lines indicate fits to a stretched-exponential function describing the long-time decay.

increasing $\gamma_{0}$, the long-time decay occurs earlier, indicating that the gel plastically yields, i.e., irreversibly rearranges, within increasingly shorter times.

The long-time decay of the echo correlation functions $g^{(2)}(m T)-1$ was fitted using a stretched-exponential function. The average relaxation time $\langle\tau\rangle=\tau \Gamma(1 / \beta) / \beta$ of the long-time decay was calculated based on the fitted relaxation time $\tau$ and its distribution determined by the stretching exponent $\beta$ (Fig. 10), with $\Gamma$ the gamma function. With increasing $\gamma_{0},\langle\tau\rangle$ decreases, indicating faster shear-induced particle rearrangements. The relaxation time $\langle\tau\rangle$ also decreases with increasing frequency $f$ and decreasing polymer concentration $c_{\mathrm{p}} / c^{*}$, except for $f=70 \mathrm{~Hz}$ and $\gamma_{0} \gtrsim 12 \%$ which might be partly due to wall slip.

In addition to the time scale of the long-time decay, quantified by $\langle\tau\rangle$, the extent of the initial decay is characterized, considering the decay to the first echo at $\Delta t=T$. The decay is quantified by the ratio $P(T) / P_{0}(T)$ [Eq. (10)] (Fig. 11), i.e., the ratio between the correlation function under shear and its value in the quiescent state, both at time $T$. A reduction in $P(T) / P_{0}(T)$ can be caused by two factors: A decrease of the nonergodic plateau and/or an acceleration of the long-time decay under shear, which both reflect shear-induced plastic rearrangements. At small strains, the value of $P(T) / P_{0}(T)$ remains approximately constant, indicating that shear does not affect significantly the nonergodic plateau. This suggests that, for these values of the strain, particles do not release their bonds and remain localized. This is different to the case of repulsive glasses, which show a decrease of the nonergodic plateau with increasing strain indicating a loosening of the cage [Petekidis et al. (2002a)]. For $\gamma_{0}>5 \%$, instead, $P(T) / P_{0}(T)$ starts to decrease similarly to the relaxation time $\langle\tau\rangle$. This suggests that, at these strains, the reduction of the first echo peak is mainly due to the acceleration of the long-time decay. The decay, and hence the 


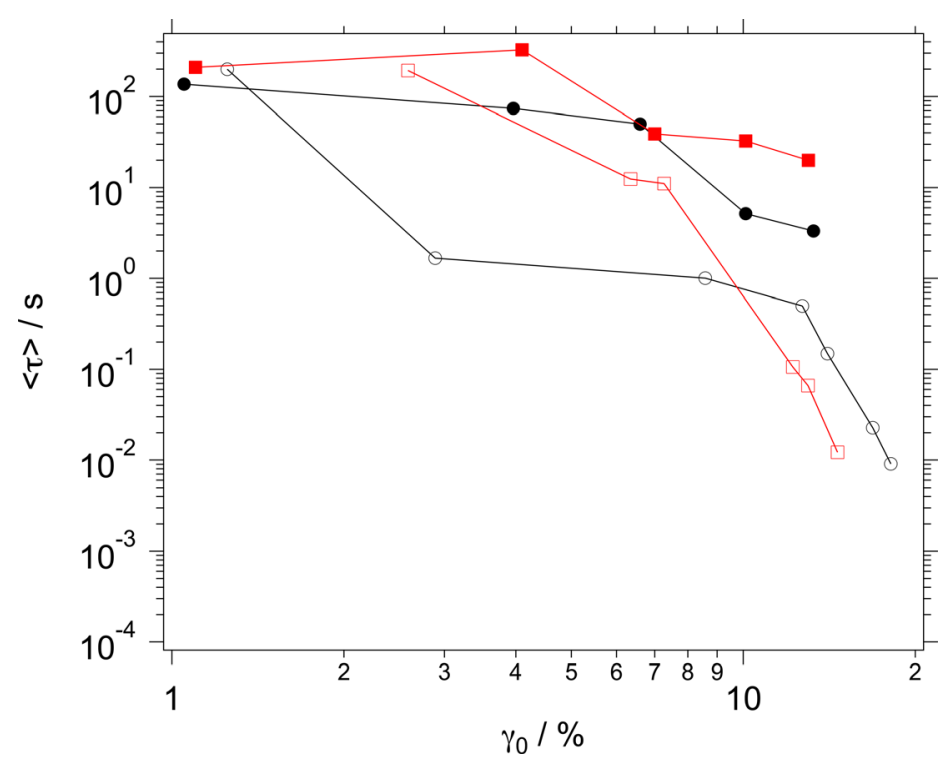

FIG. 10. Average relaxation times $\langle\tau\rangle$ as a function of strain amplitude $\gamma_{0}$ obtained by fits of the long-time decays of the LS-Echo correlation functions by a stretched-exponential function for PMMA-PS samples with polymer concentration $c_{\mathrm{p}} / c^{*}=0.5$ and frequencies $f=11 \mathrm{~Hz}(\bullet)$ and $70 \mathrm{~Hz}(\circ)$, and $c_{\mathrm{p}} / c^{*}=1.0$ and frequencies $f=11 \mathrm{~Hz}(\mathbf{\square})$ and $70 \mathrm{~Hz}(\square)$.

plastic rearrangements, are more pronounced for the smaller polymer concentration $c_{\mathrm{p}} / c^{*}=0.5$ at low frequency $f$ and the larger polymer concentration $c_{\mathrm{p}} / c^{*}=1.0$ at the high $f$. Hence, the gel with $c_{\mathrm{p}} / c^{*}=1.0$ becomes more brittle with increasing $\gamma_{0}$ at the high $f$, similar to the result of $\langle\tau\rangle$.

The faster decay of the echoes with increasing interparticle attractions resembles the findings for hard-sphere glasses upon increasing the volume fraction [Petekidis et al.

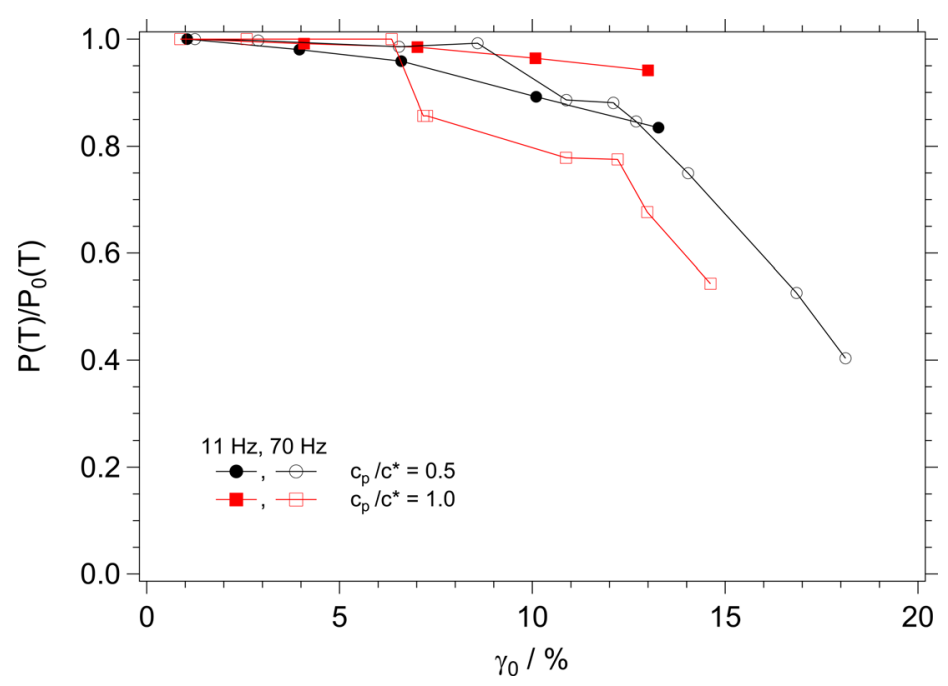

FIG. 11. Shear-induced decay of the first echo, $P(T) / P_{0}(T)$, [Eq. (10)] as a function of strain amplitude $\gamma_{0}$ for PMMA-PS samples with polymer concentration $c_{\mathrm{p}} / c^{*}=0.5$ and frequencies $f=11 \mathrm{~Hz}(\bullet)$ and $70 \mathrm{~Hz}(\circ)$, and $c_{\mathrm{p}} / c^{*}=1.0$ and frequencies $f=11 \mathrm{~Hz}(\mathbf{\square})$ and $70 \mathrm{~Hz}(\square)$. 
(2002a)]. This suggests that solids with higher elastic moduli yield more abruptly. Note though that for glasses the more abrupt yielding was mainly associated with the reduction of the free volume available for deformation when approaching random close packing.

The frequency dependence of $\langle\tau\rangle$ and $P(T) / P_{0}(T)$ differs from that of colloidal glasses [Petekidis et al. (2002a)], where Brownian motion is less efficient in relaxing the structure at higher oscillation frequencies. In addition, in a previous rheological study on similar gels [Laurati et al. (2011)], and in the rheological measurements presented here, we also found the opposite behavior, namely, an increasing yield strain with increasing frequency, although for smaller oscillation frequencies than probed here. This suggests that the different trends are due to different frequency regimes. In previous work [Smith et al. (2007); Laurati et al. (2009); Laurati et al. (2011)], we presented a model to estimate the average time needed to break a bond under oscillatory shear. It is approximated by the escape time of a particle from the interparticle potential well of depth $U_{0}$ (Table I) [Kramers (1940); Smith et al. (2007); Laurati et al. (2009); Laurati et al. (2011); Conrad et al. (2010)]. In the quiescent state, the attempt rate is determined by Brownian motion and can be estimated as $D_{\mathrm{s}} / \delta^{* 2}$ with the effective range of the potential $\delta^{*}=2 \xi^{*} R$ (see Table I for values of $\xi^{*}$ ) and the short-time self-diffusion coefficient of a particle in a cluster $D_{\mathrm{s}}=0.034 D_{0}$ based on the fits to the initial decay of $g^{(2)}(\Delta t)-1$, with the free diffusion coefficient $D_{0}$. Under shear, the attempt rate is either dominated by Brownian motion, when $D_{\mathrm{s}} / \delta^{* 2}>f$, or by the frequency of oscillation, $f$. If the attempt rate is determined by Brownian motion, escape and hence yielding become more difficult with increasing frequency due to the reduced residence time at the maxima, i.e., close to the edge of the potential well [Laurati et al. (2009); Laurati et al. (2011)]. Conversely, if the attempt rate is determined by the oscillation frequency, yielding is easier at larger frequency. The observed dependence of our gels at $f=70 \mathrm{~Hz}$, namely, lower relaxation rate $\langle\tau\rangle$ and more pronounced decay (smaller $P(T) / P_{0}(T)$ ) for a higher frequency, suggest that the rate of bond breaking is dominated by the frequency $f$. According to the model, this regime is expected for $f>D_{\mathrm{s}} / \delta^{* 2} \approx 140 \mathrm{~Hz}$, which is larger than $70 \mathrm{~Hz}$. Nevertheless, it is conceivable that the model overestimates the Brownian attempt rate, for example, because of uncertainty in the fitted value of $D_{\mathrm{s}}$, or in the determination of the effective polymer-colloid size ratio $\xi^{*}$ by the GFVT.

\section{E. Comparison of LAOS and LS-Echo results}

The results of the LAOS and LS-Echo measurements can provide a link between the macroscopic mechanical response of the gels and their microscopic dynamics under shear. The different analysis methods applied to the LAOS data consistently indicate an onset of nonlinearity, i.e., plastic deformation, at strain amplitudes $\gamma_{0} \geq 1 \%$ for $\omega=1 \mathrm{rad} / \mathrm{s}$. In comparison, LS-Echo suggests shear-induced irreversible particle rearrangements for $\gamma_{0}>1 \%$ at $f=11 \mathrm{~Hz}(\omega=69 \mathrm{rad} / \mathrm{s})$ and $\gamma_{0}>5 \%$ at $f=70 \mathrm{~Hz}(440 \mathrm{rad} / \mathrm{s})$. These results are consistent with our finding that both the onset of plastic deformation and the yield strain at small to intermediate frequencies increase with increasing frequency. This indicates that the plastic mechanical response is linked to shear-induced irreversible particle rearrangements. Note that the absence of Echo-DWS data for $1 \%<\gamma_{0}<4 \%$ does not allow us to precisely locate the onset of irreversible motion at $11 \mathrm{~Hz}$.

Furthermore, the LAOS measurements show a gradual fluidization of the system for strain amplitudes $\gamma_{0} \gtrsim 1 \%$. In the LS-Echo measurements, this is reflected in the more pronounced irreversible plastic rearrangements and the decrease of their relaxation time $\langle\tau\rangle$ with increasing $\gamma_{0}$. Therefore, fluidization seems associated with a shear-induced acceleration of the particle dynamics allowing for a reorganization of the microscopic 
structure. However, in contrast to the LAOS analysis, based on the LS-Echo measurements, the in-cycle responses cannot be identified. They can only be revealed by determining changes in the microstructure and dynamics within a cycle of deformation, as done in simulations of colloidal glasses [Koumakis et al. (2013a)]. These changes could possibly be observed using confocal microscopy under shear.

We should note here that a more quantitative link between the onset of nonlinearities in LAOS and irreversible rearrangements in LS-Echo measurements requires to investigate the same frequency regimes with the two techniques. This was not possible in this study due to wall slip at low frequencies in LS-Echo and limitations of the rheometer at high frequencies in LAOS [Poulos et al. (submitted)].

\section{CONCLUSIONS}

We investigated the yielding of colloid-polymer gels with an intermediate volume fraction under LAOS, using a combination of rheology and LS-Echo measurements. The gels start to plastically deform already at strain amplitudes $\gamma_{0} \approx 1 \%$. This is consistently indicated by the different methods used to analyze the LAOS measurements and is confirmed at a microscopic level by LS-Echo data. Furthermore, the onset of plasticity is also correctly captured by the average rheological response of the system, represented by the viscoelastic moduli obtained in the first harmonic approximation. The gel sample with stronger interparticle attractions $\left(c_{\mathrm{p}} / c^{*}=1.0\right)$ and a larger elastic modulus starts to yield at larger values of $\gamma_{0}$. At the same time, the LS-Echo measurements show that irreversible particle rearrangements increase faster with increasing the strain amplitude than those of the sample with weaker interparticle attractions. This indicates that stronger gels yield more abruptly, i.e., they are more brittle, similar to colloidal glasses at increasingly larger volume fractions [Petekidis et al. (2002a)].

Two different regimes of shear-induced restructuring are observed depending on the oscillation frequency. At small to moderate frequencies, the onset of the nonlinear response regime and the yielding process shift to larger values of $\gamma_{0}$ with increasing frequency. In contrast, at large frequencies, accessed in LS-Echo, the opposite trend is observed. This is consistent with a simple model which describes bond breaking as escape of a particle from the potential well of its nearest neighbor. The model predicts that, at small frequencies, the probability of bond breaking depends on Brownian motion and therefore decreases with increasing frequency since a particle spends less time in the vicinity of the maximum deformation where an escape is most favored. Instead, at large frequencies, the probability of bond breaking depends on the oscillation frequency and hence increases with increasing frequency.

The LAOS measurements also indicate that the in-cycle yielding is characterized by different regimes, which can be linked to the restructuring of the gel network. At small strain amplitudes $\gamma_{0}$ shear thickening and strain hardening are observed. In this regime, intercluster bonds are expected to break and hence energy is dissipated, consistent with the increase of viscosity associated with shear thickening. This disruption of the gel network might lead to stronger cluster structures, explaining the simultaneous strain hardening. With increasing $\gamma_{0}$, hence shear thickening turns into shear thinning, while strain hardening remains. Both decrease with increasing $\gamma_{0}$, indicating that the deformation at increasingly larger amplitudes leads to a progressive fluidization of the system. This is consistent with the increasingly faster relaxation of the echo correlation functions as $\gamma_{0}$ increases. The anharmonic stress analysis, which takes into account all higher harmonics of the LAOS data, suggests that strain hardening dominates over shear thinning for all $\gamma_{0}$. 
This result, which differs from other analysis methods which only retain the third harmonic contribution in the nonlinear response regime, might indicate the importance of considering all anharmonic contributions to the stress response in the analysis of LAOS data.

\section{ACKNOWLEDGMENTS}

The authors thank N. Koumakis and A. Poulos for stimulating discussions, A. Pamvouxoglou for help during the initial stages of the experiments at FORTH, and A. B. Schofield for providing the PMMA particles. They are also grateful to Professor R. Ewoldt for providing the program MITLaos, which was used to analyze the LAOS data by expanding the stress into Chebyshev polynomials. The authors acknowledge EU funding through the Infrastructure Project "ESMI" (CP\&CSA-2010-262348) and the SoftComp network, and support by the Deutsche Forschungsgemeinschaft (DFG).

\section{References}

Aarts, D. G. A. L., R. Tuinier, and H. N. W. Lekkerkerker, "Phase behaviour of mixtures of colloidal spheres and excluded-volume polymer chains,” J. Phys.: Condens. Matter 14, 7551-7561 (2002).

Asakura, S., and F. Oosawa, "Interaction between two bodies immersed in a solution of macromolecules," J. Chem. Phys. 22, 1255-1256 (1954).

Ballesta, P., N. Koumakis, R. Besseling, W. C. K. Poon, and G. Petekidis, "Slip of gels in colloid-polymer mixtures under shear," Soft Matter 9, 3237-3245 (2013).

Bergenholtz, J., W. C. K. Poon, and M. Fuchs, "Gelation in model colloid-polymer mixtures," Langmuir 19, 4493-4503 (2003).

Berry, G. C., "Thermodynamic and conformational properties of polystirene. I. Light-scattering studies on dilute solutions of linear polystirenes," J. Chem. Phys. 44, 4550 (1966).

Brader, J. M., M. Siebenbürger, M. Ballauff, K. Reinheimer, M. Wilhelm, S. J. Frey, F. Weysser, and M. Fuchs, "Nonlinear response of dense colloidal suspensions under oscillatory shear: Mode-coupling theory and Fourier transform rheology experiments," Phys. Rev. E 82, 061401 (2010).

Carrier, V., and G. Petekidis, "Nonlinear rheology of colloidal glasses of soft thermosensitive microgel particles," J. Rheol. 53, 245-273 (2009).

Cho, M., K. Hyun, K. Ahn, and S. Lee, "A geometrical interpretation of large amplitude oscillatory shear response," J. Rheol. 49, 747-758 (2005).

Colombo, J., A. Widmer-Cooper, and E. Del Gado, "Microscopic picture of cooperative processes in restructuring gel networks,” Phys. Rev. Lett. 110, 198301 (2013).

Conrad, J. C., H. M. Wyss, S. Manley, V. Trappe, K. Miyazaki, L. J. Kaufman, A. Schofield, D. R. Reichman, and D. A. Weitz, "Arrested fluid-fluid phase separation in depletion systems: Implications of the characteristic length on gel formation and rheology," J. Rheol. 54, 421-438 (2010).

Daniel, C., I. W. Hamley, M. Wilhelm, and W. Mingvanish, "Non-linear rheology of a face-centred cubic phase in a diblock copolymer gel," Rheol. Acta 40, 39-48 (2001).

Derakhshandeh, B., G. Petekidis, S. S. Sabet, W. Y. Hamad, and S. G. Hatzikiriakos, “Ageing, yielding, and rheology of nanocrystalline cellulose suspensions," J. Rheol. 57, 131-148 (2013).

Dibble, C. J., M. Kogan, and M. J. Solomon, "Structure and dynamics of colloidal depletion gels: Coincidence of transitions and heterogeneity," Phys. Rev. E 74, 041403 (2006).

Eberle, A. P. R., N. J. Wagner, and R. Castaneda-Priego, "Dynamical arrest transition in nanoparticle dispersions with short-range interactions," Phys. Rev. Lett. 106, 105704 (2011).

Ewoldt, R. H., P. Winter, J. Maxey, and G. H. McKinley, "Large amplitude oscillatory shear of pseudoplastic and elastoviscoplastic materials," Rheol. Acta 49, 191-212 (2010).

Ewoldt, R. H., A. E. Hosoi, and G. H. McKinley, "New measures for characterizing nonlinear viscoelasticity in large amplitude oscillatory shear,” J. Rheol. 52, 1427-1458 (2008). 
Fleer, G. J., and R. Tuinier, “Analytical phase diagram for colloid-polymer mixtures,” Phys. Rev. E, 76, 041802 (2007).

Fuchs, M., and K. S. Schweizer, "Structure of colloid-polymer suspensions," J. Phys.: Condens. Matter 14, R239-R269 (2002).

Furst, E. M., and J. P. Pantina, "Yielding in colloidal gels due to nonlinear microstructure bending mechanics," Phys. Rev. E 75, 050402 (2007).

Hébraud, P., F. Lequeux, J. P. Munch, and D. J. Pine, "Yielding and rearrangements in concentrated emulsions," Phys. Rev. Lett. 78, 4657 (1997).

Höler, S., S. Cohen-Addad, and H. Hoballah, "Periodic nonlinear bubble motion in aqueous foam under oscillating shear strain," Phys. Rev. Lett. 79, 1154 (1997).

Hyun, K., M. Wilhelm, C. O. Klein, K. S. Cho, J. G. Nam, K. H. Ahn, S. J. Lee, R. H. Ewoldt, and G. H. McKinley, "A review of nonlinear oscillatory shear tests: Analysis and application of large amplitude oscillatory shear (LAOS)," Prog. Polym. Sci. 36, 1697-1753 (2011).

Koumakis, N., Ph.D. thesis, University of Crete, Heraklion, 2011.

Koumakis, N., and G. Petekidis, "Two step yielding in attractive colloids: Transition from gels to attractive glasses," Soft Matter 7, 2456-2470 (2011).

Koumakis, N., A. Pamvouxoglou, A. S. Poulos, and G. Petekidis, "Direct comparison of the rheology of model hard and soft particle glasses," Soft Matter 8, 4271-4284 (2012).

Koumakis, N., J. F. Brady, and G. Petekidis, "Complex oscillatory yielding of model hard-sphere glasses," Phys. Rev. Lett. 110, 178301 (2013a).

Koumakis, N., P. Ballesta, R. Besseling, W. C. K. Poon, J. F. Brady, and G. Petekidis, "Colloidal gels under shear: Strain rate effects," AIP Conf. Proc. 1518, 365 (2013b).

Kramers, H. A., "Brownian motion in a field of force and the diffusion model of chemical reactions," Physica A 7, 284-304 (1940).

Laeueger, J., and H. Stettin, "Differences between stress and strain control in the non-linear behaviour of complex fluids,” Rheol. Acta 49, 909-930 (2010).

Langela, M., U. Wiesner, H. Spiess, and M. Wilhelm, "Microphase reorientation in block-copolymer melts as detected via FT rheology and 2D SAXS,” Macromolecules 35, 3198-3204 (2002).

Laurati, M., G. Petekidis, N. Koumakis, F. Cardinaux, A. B. Schofield, J. M. Brader, M. Fuchs, and S. U. Egelhaaf, "Structure, dynamics, and rheology of colloid-polymer mixtures: From liquids to gels," J. Chem. Phys. 130, 134907 (2009).

Laurati, M., S. U. Egelhaaf, and G. Petekidis, "Non-linear rheology of colloidal gels with intermediate volume fraction," J. Rheol. 55, 673-706 (2011).

Le Grand, A., and G. Petekidis, "Effects of particle softness on the rheology and yielding of colloidal glasses," Rheol. Acta 47, 579-590 (2008).

Lee, M. H., and E. M. Furst, "Response of a colloidal gel to a microscopic oscillatory strain," Phys. Rev. E 77, 041408 (2008).

Lekkerkerker, H. N. W., W. C. K. Poon, P. N. Pusey, A. Stroobants, and P. B. Warren, "Phase-behavior of colloid plus polymer mixtures," Europhys. Lett. 20, 559-564 (1992).

Lu, P. J., E. Zaccarelli, F. Ciulla, A. B. Schofield, F. Sciortino, and D. A. Weitz, "Gelation of particles with short-range attraction," Nature 453, 499-503 (2008).

Neidhofer, T., M. Wilhelm, and B. Debbaut, "Fourier-transform rheology experiments and finite-element simulations on linear polystyrenesolutions," J. Rheol. 47(6), 1351-1371 (2003).

Petekidis, G., A. Moussaid, and P. N. Pusey, "Rearrangements in hard-sphere glasses under oscillatory shear strain,” Phys. Rev. E 66, 051402 (2002a).

Petekidis, G., D. Vlassopoulos, and P. N. Pusey, "Yielding and flow of colloidal glasses," Faraday Discuss. 123, 287-302 (2003).

Petekidis, G., P. N. Pusey, A. Moussaid, S. U. Egelhaaf, and W. C. K. Poon, "Shear-induced yielding and ordering in concentrated particle suspensions," Physica A 306, 334-342 (2002b).

Pham, K. N., G. Petekidis, D. Vlassopoulos, S. U. Egelhaaf, P. N. Pusey, and W. C. K. Poon, "Yielding of colloidal glasses,” Europhys. Lett. 75, 624-630 (2006). 
Pham, K. N., G. Petekidis, D. Vlassopoulos, S. U. Egelhaaf, W. C. K. Poon, and P. N. Pusey, "Yielding behavior of repulsion- and attraction-dominated colloidal glasses," J. Rheol. 52, 649-676 (2008).

Pham, K. N., S. U. Egelhaaf, A. Moussaid, and P. N. Pusey, "Ensemble-averaging in dynamic light scattering by an echo technique," Rev. Sci. Instrum. 75, 2419 (2004).

Poon, W. C. K., “The physics of a model colloid-polymer mixture,” J. Phys.: Condens. Matter 14, R859-R880 (2002).

Poulos, A. S., F. Renou, N. Koumakis, and G. Petekidis, "Large amplitude oscillatory shear (LAOS) in model colloidal suspensions and glasses: Frequency dependence and resonance effects," Rheol. Acta (submitted).

Poulos, A. S., J. Stellbrink, and G. Petekidis, "Flow of concentrated solutions of starlike micelles under largeamplitude oscillatory shear," Rheol. Acta 52, 785-800 (2013).

Renou, F., J. Stellbrink, and G. Petekidis, "Yielding processes in a colloidal glass of soft star-like micelles under large amplitude oscillatory shear (LAOS),” J. Rheol. 54, 1219-1243 (2010).

Rogers, S. A., B. M. Erwin, D. Vlassopoulos, and M. Cloitre, "A sequence of physical processes determined and quantified in LAOS: application to a yield stress fluid," J. Rheol. 55, 435-458 (2011).

Schaertl, W., and H. Silescu, "Brownian dynamics of polydisperse colloidal hard-spheres-equilibrium structures and random close packings," J. Stat. Phys. 77, 1007-1025 (1994).

Schlatter, G., G. Fleuary, and R. Muller, "Fourier transform rheology of branched polyethylene:experiments and models for assessing the macromolecular architecture," Macromolecules 38, 6492-6503 (2005).

Shah, S. A., S. Ramakrishnan, Y. L. Chen, K. S. Schweizer, and C. F. Zukoski, "Microstructure of dense colloid-polymer suspensions and gels," J. Phys.: Condens. Matter 15, 4751 (2003a).

Shah, S. A., Y. L. Chen, and K. S. Schweizer, "Phase behavior and concentration fluctuations in suspensions of hard spheres and nearly ideal polymers," J. Chem. Phys. 118, 3350-3361 (2003b).

Shah, S. A., Y. L. Chen, K. S. Schweizer, and C. F. Zukoski, "Viscoelasticity and rheology of depletion flocculated gels and fluids," J. Chem. Phys. 119, 8747-8760 (2003c).

Smith, P. A., G. Petekidis, S. U. Egelhaaf, and W. C. K. Poon, "Yielding and crystallization of colloidal gels under oscillatory shear," Phys. Rev. E 76, 041402 (2007).

Valadez-Pérez, N. E., Y. Liu, A. P. R. Eberle, N. J. Wagner, and R. Castaneda-Priego, "Dynamical arrest in adhesive hard-sphere dispersions driven by rigidity percolation,”. Phys. Rev. E 88, 060302 (2013).

Varadan, P., and M. J. Solomon, "Direct visualization of long-range heterogeneous structure in dense colloidal gels," Langmuir 19, 509-512 (2003).

Verduin, H., and J. K. G. Dhont, "Phase-diagram of a model adhesive hard-sphere dispersion,” J. Colloid Interface Sci. 172 425-437 (1995).

Verhaegh, N. A. M., D. Asnaghi, H. N. W. Lekkerkerker, M. Giglio, and L. Cipelletti, "Transient gelation by spinodal decomposition in colloid-polymer mixtures," Physica A 242, 104-118 (1997).

Wilhelm, M., D. Maring, and H. Spiess, "Fourier-transform rheology," Rheol. Acta 37, 399-405 (1998).

Wilhelm, M., P. Reinheimer, and M. Ortseifer, "High sensitivity Fourier-transform rheology," Rheol. Acta 38, 349-356 (1999).

Wilhelm, M., P. Reinheimer, M. Ortseifer, T. Neidhofer, and H. Spiess, "The crossover between linear and nonlinear mechanical behaviour in polymer solutions as detected by Fourier-transform rheology," Rheol. Acta 39, 241-246 (2000).

Zaccarelli, E., "Colloidal gels: Equilibrium and nonequilibrium routes," J. Phys.: Condens. Matter 19, 323101 (2007).

Zaccone, A., and E. Del Gado, "Elasticity of arrested short-ranged attractive colloids: Homogeneous and heterogeneous glasses,” Phys. Rev. Lett. 103, 208301 (2009). 